\title{
Экспериментальное исследование
} магнитных свойств жидкого кристалла МВВА и ферромагнитных суспензий на его

\section{основе}

\section{О. О. Пермякова, В. А. Попов, В. Г. Гилев}

Пермский государственный национальный исследовательский университет 614990, Пермь, ул. Букирева, 15

email: psksvs@yandex.ru

\begin{abstract}
Приведены результаты сравнительных исследований магнитного перехода Фредерикса нематического жидкого кристалла МВВА (НЖК) и ферронематической суспензии (ФНС) на его основе. В качестве магнитных частиц использовались частицы магнетита $\left(\mathrm{Fe}_{3} \mathrm{O}_{4}\right)$, объемная доля которых составляла $f=4 \cdot 10^{-4}$. Методами электронной микроскопии показано, что частицы магнетита имеют квазисферическую форму. Построено распределение частиц по размерам. Средний диаметр частиц составил 10 нм. Описаны методика подготовки образцов при допировании нематической матрицы ферромагнитными частицами и устройство жидкокристаллической ячейки. Диэлектрические измерения выполнены на прецизионном анализаторе RLC WK 6505B в постоянном магнитном поле с индукцией от 0 до 0.6 Тл. Показано, что пороговое поле Фредерикса для ФНС значительно меньше, чем для нематической матрицы. Построены температурные зависимости порогового поля Фредерикса. Показано, что с ростом температуры значения порогового поля убывают.
\end{abstract}

\section{Experimental investigation of the magnetic properties of a liquid crystal MBBA and ferromagnetic suspensions based on it}

\author{
O. O. Permyakova, V. A. Popov, V. G. Gilev
}

Perm State University, Bukireva St. 15, 614990, Perm

email: psksvs@yandex.ru

This article shows the measured dependences of the Frederiksz threshold field for liquid crystal (LCD) MBBA and ferromagnetic suspension (FN) based on it. Magnetite particles (Fe3O4) were used as magnetic particles, the volume fraction of which was $f=4 \cdot 10^{-4}$. Electron microscopy showed that magnetite particles have a quasispherical shape. The particle size distribution is constructed. The average particle diameter was $10 \mathrm{~nm}$. A technique for preparing samples for doping a nematic matrix with ferromagnetic particles and a liquid crystal cell device are described. Dielectric measurements are performed on a precision RLC WK 6505B analyzer in a constant magnetic field with induction from 0 to $0.6 \mathrm{~T}$. It is shown that the Frederiksz threshold field for the FN is significantly smaller than for the nematic matrix. Temperature dependences of the Frederiksz 
threshold field are constructed. It is shown that with a temperature rise the threshold field values decrease.

Keywords: Fréedericksz transition; liquid crystal; ferronematic; electron microscopy

Received 02.11.2017; accepted 02.11.2017

doi: 10.17072/1994-3598-2017-3-60-65

\section{1. Введение}

Жидкие кристаллы - это вещества, обладающие одновременно свойствами и жидкостей, и кристаллов. По своим механическим свойствам они похожи на жидкости, а основные физические свойства: оптические, электрические, магнитные и другие, как у твердых кристаллических веществ.

Молекулы в жидких кристаллах могут быть упакованы по-разному. В зависимости от упаковки различают три основных типа структур ЖК: нематический (нематики), смектический (смектики) и холестерический (холестерики). У нематических жидких кристаллов длинные оси молекул ориентированы только в одном измерении, но при этом центры тяжести молекул расположены в пространстве хаотично.

Для описания ориентационной структуры ЖК используется единичный вектор $\mathbf{n}$, называемый директором, который отражает преимущественное расположение длинных осей молекул. Изменять ориентационную структуру можно с помощью внешних силовых полей (механических, электрических, магнитных). Пороговое изменение направления директора под действием внешнего магнитного или электрического поля носит название перехода Фредерикса, а само поле называется критическим или пороговым полем Фредерикса [1].

Как правило, магнитная восприимчивость индивидуальных НЖК не велика и, как следствие, критическое поле Фредерикса имеет большую величину $\sim 1$ Тл. Для увеличения магнитной восприимчивости НЖК его допируют ферромагнитными частицами. Система, состоящая из нематика с взвешенными в нем ферромагнитными гранулами, называется ферронематиком. Теории ферромагнитных суспензий в жидких кристаллах посвящена фундаментальная работа Ф. Брошара и П. де Жена [2].

Как показывает анализ экспериментальных и теоретических работ, связанных с изучением свойств ФНС [3-7], даже незначительная добавка магнитных частиц приводит к сильному изменению физических свойств такой суспензии. При этом влияние оказывает как материал частиц [4], так и их форма [5], а также энергия сцепления частиц с ЖК-матрицей и их взаимное расположение (планарное или гомеотропное) [6]. Немаловажную роль играет и седиментационная устойчивость
ФНС. Система однородна в магнитном поле только в том случае, когда выполнятся неравенство $\mu H / k T<1$, где $\mu$ - магнитный момент ферромагнитной частицы; $H$ - напряженность магнитного поля; $k$ - постоянная Больцмана; $T$ - температура. Если $\mu H / k T>1$, наступает явление сегрегации гранул, в результате чего формируются зоны коагулированных гранул и зоны с повышенной концентрацией ферромагнитных частиц. Процессы коагуляции зависят от методики приготовления ФНС. Определенные проблемы возникают при измерении концентрации дисперсных частиц. Так, в работе [7] концентрация частиц рассчитывалась как отношение количества изначально добавленной массы магнетита к массе несущего ЖК. В действительности реальная концентрация частиц, как правило, меньше, так как часть гранул может не сцепляться с ЖК матрицей. Помимо этого, в процессе допирования матрицы определенная доля частиц объединяется в агрегаты, которые в магнитном поле непременно будут седиментировать.

В настоящей работе проведены тестовые измерения температурной зависимости магнитного перехода Фредерикса нематического жидкого кристалла МВВА и ферронематической суспензии на его основе. При подготовке ФНС применен широко используемый для магнитных жидкостей метод магнитной сепарации, что позволяет до проведения экспериментов удалить агрегаты частиц и повысить седиментационную устойчивость образцов.

\section{2. Объект исследования}

В данной работе в качестве нематической матрицы использовался жидкий кристалл МВВА: 4метоксибензилиден-4'-бутиланилин $\left(\mathrm{C}_{18} \mathrm{H}_{21} \mathrm{NO}\right)$, а в качестве примесных магнитных частиц - частицы магнетита $\left(\mathrm{Fe}_{3} \mathrm{O}_{4}\right)$. Коллоидные частицы получены осаждением из раствора магнитной жидкости: магнетит в керосине, стабилизированной олеиновой кислотой.

В процессе приготовления ФНС феррочастицы, покрытые стабилизатором, смешивались с МВВА. Контейнер со смесью помещался в рабочую полость ультразвуковой ванны Digital Ultrasonic Cleaner CD 4820, где в области изотропной фазы MBBA при температуре $50-60^{\circ} \mathrm{C}$ смесь перемешивалась до получения однородного раствора.

Для удаления не связавшихся с матрицей ЖК магнитных частиц и их агрегатов раствор поме- 
щался в градиентное магнитное поле, где выдерживался в течение $\approx 24$ час. Крупные и несвязанные частицы оседали на дно пробирки. В опытах использовался только верхний слой суспензии («легкая фракция»).

Объемная доля $\mathrm{Fe}_{3} \mathrm{O}_{4}$ «легкой фракции» измерялась с помощью пикнометра объемом $5 \mathrm{~cm}^{3}$, помещенного в термостат. Измерения массы МВВА и ферронематической смеси выполнены с помощью аналитических весов ЛВ-210А 2-го класса точности, абсолютная погрешность измерения которых составляла \pm 0.4 мг.

Расчет объемной доли частиц производился по формуле

$$
\varphi=\frac{\rho_{\Phi H C}-\rho_{H ж K}}{\rho_{M A \Gamma}-\rho_{H ж K}},
$$

где $\rho_{\text {ФнС }}, \rho_{\text {мАГ }}$ и $\rho_{\text {нжк }}-$ плотности ферромагнитной смеси, нематической матрицы и магнетита, соответственно. Объемная доля частиц исследуемого образца ФНС составила $4 \cdot 10^{-4}$.

\section{3. Измерительная ячейка}

Жидкокристаллические ячейки являются основным элементом любого устройства, основанного на данном типе материалов. Поэтому их качество играет ключевую роль при использовании как в фундаментальных исследованиях, так и в практических приложениях.

В опытах использовались ячейки типа «сэндвич», состоящие из двух стеклянных подложек, слоя ориентанта и прокладок, задающих толщину слоя ЖК.

Стеклянные подложки для ЖК ячеек вырезались из стеклянного листа, покрытого проводящим слоем ITO. Геометрические размеры подложек составляли $20 \times 25$ мм. Положение электродов и рабочая область в центре подложек размерами $10 \times 10$ мм, необходимая для исключения краевых эффектов, задавались с помощью маски, в роли которой выступала липкая лента.

В дальнейшем подложки с нанесенными на них масками обрабатывались с помощью травления в смеси порошка цинка и $30 \%$-ного раствора соляной кислоты $(\mathrm{HCl})$. После процедуры травления подложки промывались в ультразвуковой ванне в несколько стадий: в растворе моющего средства и дистиллированной воде.

Далее на подложку наносился ориентант, в качестве которого использовался 10\%-ный водный раствор поливинилового спирта (ПВС). Для равномерного нанесения ориентанта подложка с капелькой ПВС помещалась в центрифугу, столик которой плавно разгонялся до частоты 3000 об/с и поддерживался в таком состоянии в течение $30 \mathrm{c}$. Высокая скорость вращения центрифуги обеспечивает равномерную толщину нанесения полимер- ного ориентанта. Для завершения процесса полимеризации стекло со слоем ПВС помещалось в сушильный шкаф, разогретый до температуры $80{ }^{\circ} \mathrm{C}$ на 30 мин. После этого поверхность натиралась ворсистой тканью в одном направлении для создания планарной ориентации ЖК в ячейке [8].

Для задания толщины слоя ЖК использовались майларовые прокладки. Прокладки зажимались между стеклами и фиксировались в специальном механическом держателе. Внешний зазор между стеклянными подложками и прокладками равномерно заполнялся светочувствительным клеем, который полимеризовался под действием ультрафиолетового излучения.

Оценка толщины слоя ЖК и угла преднаклона директора выполнялась с помощью измерений интерференции поляризованных лучей по методике работы [9]. Измерения показали, что в измерительном зазоре осуществляется планарная ориентация директора с углом преднаклона не более $3^{\circ}$. $\mathrm{B}$ используемых в опытах ячейках толщина слоя ЖК составляла 25 мкм.

\section{4. Результаты измерений}

\section{1.Электронная микроскопия}

Оценки размеров и формы частиц магнетита выполнены на низковольтном электронном микроскопе LVEM5 (DeLong Instruments). Разрешение микроскопа в режиме ТЕМ составляет 2 нм.

В процессе пробоподготовки «верхняя» фракция ФНС наносилась на металлическую сеточку и выдерживалась в сушильной камере при температуре $80{ }^{\circ} \mathrm{C}$ до полного испарения МВВА.

На рис. 1 приведен фрагмент одной из фотографий (масштаб указан на рисунке).

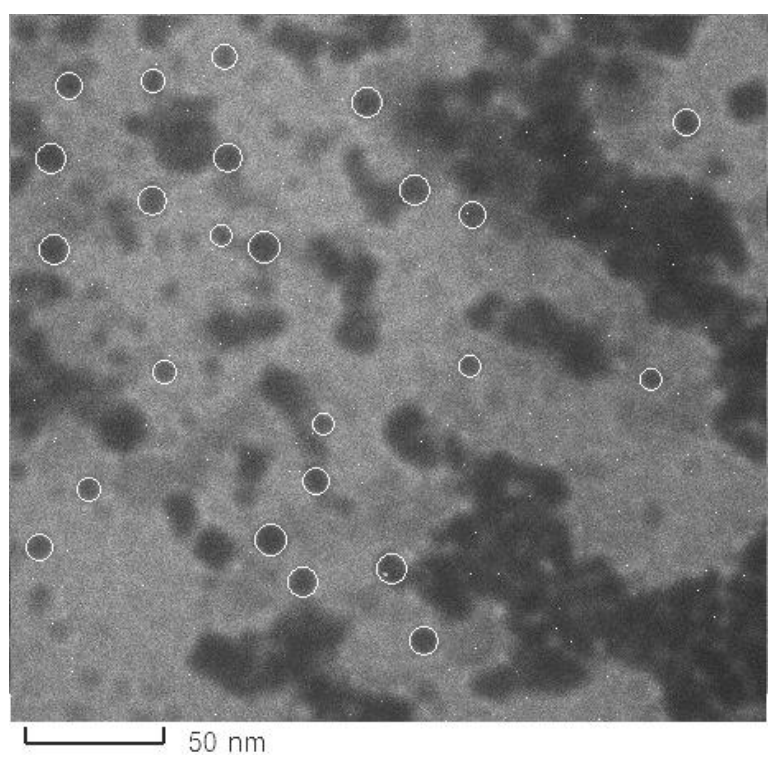

Рис. 1. К определению размера и формы частиц 
Как видно из рисунка, основная масса частиц распределена неравномерно. Образование скоплений частиц может быть обусловлено тем, что в процессе высушивания силы поверхностного натяжения приводят к агломерации частиц. Тем не менее видно, что отдельные частицы имеют почти сферическую форму.

Для определения среднего размера частиц они были аппроксимированы окружностями. Для аппроксимации использовалась программа ImageJ. На основании измеренных значений размеров частиц была построена гистограмма зависимости процентного содержания частиц в растворе от их диаметра. Результаты расчетов показаны на рис. 2.

Распределение размеров частиц в среднем подчиняется нормальному закону с наиболее вероятным значением диаметра частиц 10 нм.

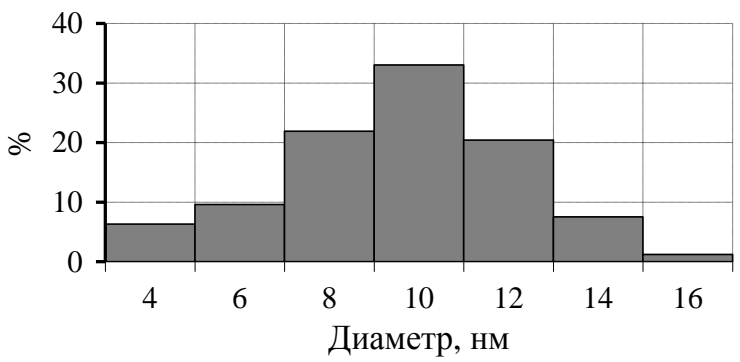

Рис. 2. Гистограмма зависимости проиентного содержания частии в растворе от их диаметра

\section{2.Эффект Фредерикса}

Существует два наиболее распространенных метода для исследования перехода Фредерикса. Это оптические методы наблюдения и макроскопические измерения диэлектрических, магнитных или тепловых свойств ЖК.

Макроскопический метод заключается в исследовании среднего состояния упорядочения молекул ЖК под действием зондирующего поля (тепловой градиент, магнитное или электрическое поля). Структурные свойства вещества определяется с помощью измерений анизотропных свойств, например, диэлектрической проницаемости, магнитной восприимчивости или теплопроводности [1]. Наиболее чувствительным из них является метод двулучепреломления, но более информативным - метод диэлектрической спектроскопии.

В настоящей работе мы использовали диэлектрический метод измерения.

Измерительная ячейка помещалась в термостатирующую оболочку, через которую прокачивалась вода от струйного ультратермостата VT-14. Температура образца контролировалась с помощью хромель-алюмелевой термопары, спай которой находился на внешней стенке ячейки, и измерялась цифровым измерителем температуры Center 306 с точностью не менее $0.1^{\circ} \mathrm{C}$. Вся система размещалась между полюсами лабораторного элек- тромагнита ФЛ-1 с размером полюсных наконечников 100 мм. Питание электромагнита осуществлялось с помощью стабилизированного источника питания постоянного тока GPR-7510HD (GW INSTEK).

Для измерения диэлектрических характеристик образцов: ёмкости и тангенса угла диэлектрических потерь был использован прецизионный анализатор RLC серии WK6505B. Такой прибор способен проводить измерения иммитанса в диапазоне частот от 20 Гц до 5 МГц с заданной амплитудой зондирующего гармонического напряжения и автоматическим сканированием по частоте.

Для исключения паразитной емкости подводящих проводов результаты измерений нормировались как:

$$
C^{*}=\frac{C-C_{\min }}{C_{\max }-C_{\min }}
$$

где $C_{\min }-$ наименьшее значение емкости; $C_{\max }-$ наибольшее значение емкости; $C$ - текущее значение емкости; $C^{*}$ - текущее значение емкости с учетом нормировки. Такая нормированная емкость $C^{*}$ изменяется в пределах от 0 до 1 . Все результаты, представленные ниже, приведены для нормированной емкости. Измерения выполнены на частоте 10 кГц.

На рис. 3 представлены результаты измерений матрицы-носителя - МВВА в зависимости от индукции магнитного поля и температуры.

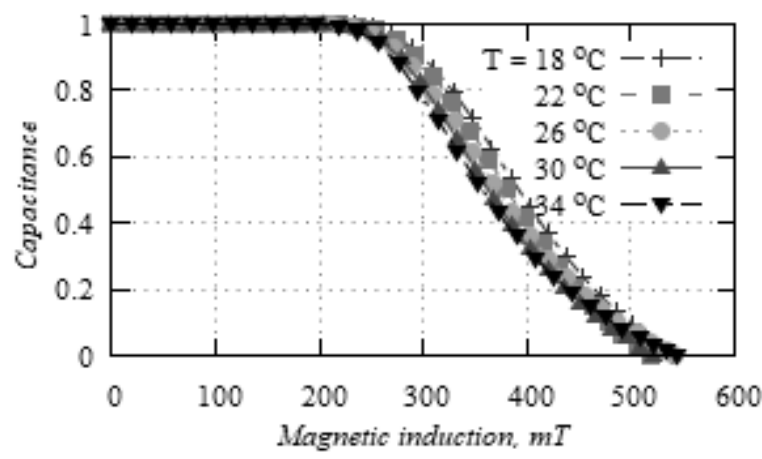

Рис. 3. Зависимость емкости ячейки, заполненной МВВА, от индукиии магнитного поля при различHolx meмnepamypax

Для определения критического поля Фредерикса обычно применяют следующий известный прием [7]: по самому крутому участку кривой $C^{*}=F(B)$ проводится линия до пересечения с прямой, отвечающей емкости ячейки, до включения внешнего магнитного поля; точка пересечения этих двух аппроксимационных кривых принимается за критическое поле Фредерикса.

MBВА обладает отрицательной диэлектрической анизотропией, поэтому после достижения порога Фредерика емкость образца при изначально планарной ориентации директора уменьшается. 
На рис. 4 показана полевая зависимость нормированной емкости ферронематической суспензии при различных температурах.

Видно, что добавка ферромагнитных частиц приводит к уменьшению критического поля перехода Фредерикса, что свидетельствует о планарном сцеплении магнитных частиц с ЖК-матрицей для выбранной геометрии эксперимента [6].

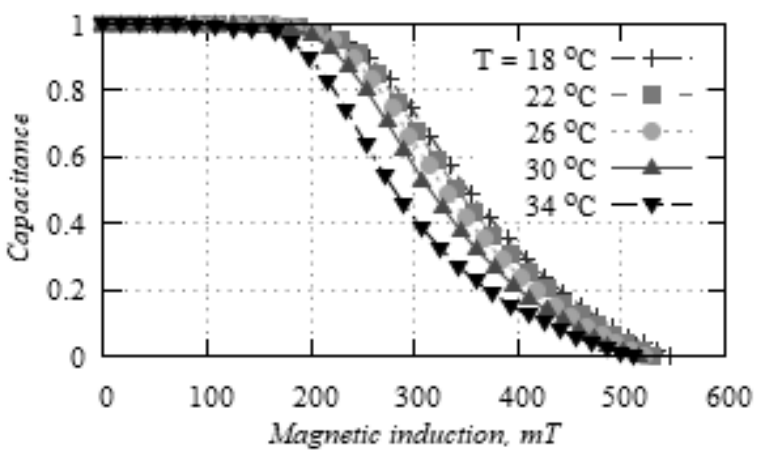

Рис. 4. Зависимость емкости ячейки, заполненной исследуемой ФНС, от индукиии магнитного поля при различных температурах

Как видно из приведенных графиков (рис. 3, 4), значение поля перехода при увеличении температуры уменьшается как для МВВА, так и для суспензии на его основе, что связано с изменением параметра порядка расположения молекул.

Сравнительные результаты изменений критического поля Фредерикса для МВВА и ФНС показаны на рис. 5. Числовые значения отражены в таблице.

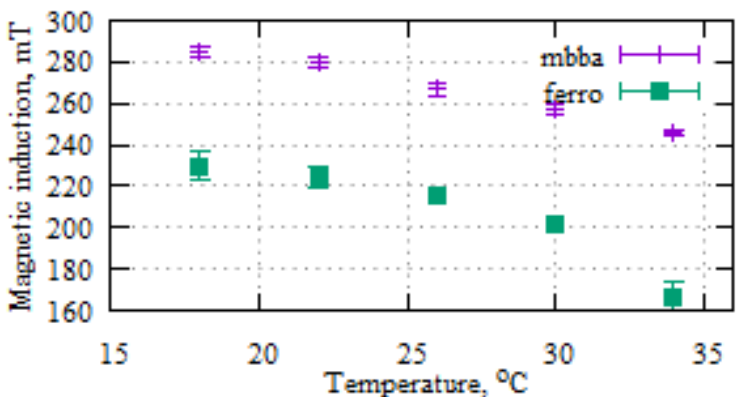

Рис. 5. Зависимость порогового поля от температуры для чистого МВВА («плюсы»)) и для суспензии на его основе («квадратыл»)

Таблица. Значения порогового поля в зависимости от температуры

\begin{tabular}{|c|c|r|r|}
\hline $\mathrm{T}\left({ }^{\circ} \mathrm{C}\right)$ & $\mathrm{B}_{\mathrm{c}}{ }^{\mathrm{mbba}}(\mathrm{mT})$ & $\mathrm{B}_{\mathrm{c}}{ }^{\text {ferro }}(\mathrm{mT})$ & $\Delta \mathrm{B}_{\mathrm{c}}(\mathrm{mT})$ \\
\hline 18 & $285 \pm 2$ & $230 \pm 7$ & 55 \\
\hline 22 & $280 \pm 3$ & $224 \pm 5$ & 56 \\
\hline 26 & $267 \pm 3$ & $215 \pm 3$ & 52 \\
\hline 30 & $257 \pm 3$ & $202 \pm 3$ & 55 \\
\hline 34 & $246 \pm 2$ & $167 \pm 7$ & 79 \\
\hline
\end{tabular}

В целом значения порогового поля по мере увеличения температуры убывают как для MBВА, так и для ферромагнитной суспензии на его основе.

Анализ диэлектрических спектров не входит в цели настоящей работы. Однако заметим, что в диапазоне частот от 100 Гц до 5 МГц максимумов мнимой части диэлектрической проницаемости не наблюдалось. Это косвенно свидетельствует об отсутствии в образцах агрегатов частиц.

\section{5. Заключение}

Проведено экспериментальное исследование магнитного перехода Фредерикса нематического жидкого кристалла МВВА (НЖК) и ферронематической суспензии (ФНС) на его основе.

Методами электронной микроскопии показано, что частицы магнетита имеют квазисферическую форму со средним диаметром частиц $10 \mathrm{Hм}$.

Методами диэлектрических измерений определены значения критического поля Фредерикса в зависимости от индукции внешнего магнитного поля и температуры среды. Показано, что при допировании нематической матрицы ферромагнитными частицами значения поля Фредерикса заметно уменьшаются. Построена зависимость критического поля Фредерикса от температуры среды. Сделан вывод о планарном сцеплении частиц с молекулами нематической матрицы.

\section{Список литературы}

1. Жен де П. Физика жидких кристаллов. М.: Мир, 1973. $400 \mathrm{c}$.

2. Brochard F., de Gennes $P$. G. Theory of magnetic suspensions in liquid crystals // Journal de Physique. 1970. Vol. 31. P. 691-708.

3. Buluy O., Ouskova E., Reznikov Yu., Litvin P. Preparation and properties of a ferro-magnetic nematic suspension // Ukranian Journal of Physics. 2004. Vol. 49. P. A48-A50.

4. Mishra M., Dabrowski R. S., Vij J. K., Mishra A., Dhar $R$. Electrical and electro-optical parameters of 4'-octyl-4-cyanobiphenyl nematic liquid crystal dispersed with gold and silver nanoparticles, Liquid Crystals, 2015. Volume 42, pages 1580-1590.

5. Kopčanský P., Tomašovičová N., Koneracká M., Závišová V., Timko M., Džarová A., Šprincová A., Éber N., Fodor-Csorba K., Tóth-Katona T., Vajda A., Jadzyn J. Structural changes in the 6CHBT liquid crystal doped with spherical, rodlike, and chainlike magnetic particles // Physical Review. 2008. Vol. 78, 011702.

6. Zakhlevnykh A. N., Petrov D. A. Weak coupling effects and re-entrant transitions in ferronematic liquid crystals // Journal of Molecular Liquids. 2014. Vol. 198. P. 223-233

7. Kopčanský P., Tomašovičová N., Tóth-Katona T., Éber N., Timko M, Závišová V., Majorošová J., Rajňak M., Jadzyn J., Chaud X. Increasing the magnetic sensitivity of liquid crystals by rod-like 
magnetic nanoparticles // Magnetohydrodynamics. 2013. Vol. 49. P. 586-591.

8. Коньяр Ж. Ориентация нематических жидких кристаллов и их смесей. Минск: Университетское, $1986.104 \mathrm{c}$.

9. Simon R., Nicholas D. V. Interferometric method of measuring tilt angles in aligned thin films of nematic liquid crystals. // Journal of Physics D: Applied Physics. 1985. Vol. 18. P. 1423-1430.

\section{References}

1. Jen de W. H. The physics of liquid crystals. Oxford Univ. Press, 1974. 333 p.

2. Brochard F., Jen de W. H. Theory of magnetic suspensions in liquid crystals. Journal de Physique, 1970, vol. 31, pp. 691-708.

3. Buluy O., Ouskova E., Reznikov Yu., Litvin P. Preparation and properties of a ferro-magnetic nematic suspension. Ukranian Journal of Physics, 2004, vol. 49. pp. A48-A50.

4. Mukesh M., Dabrowski R. S., Vij J. K., Mishra A., Dhar R. Electrical and electro-optical parameters of 4-octyl-4-cyanobiphenyl nematic liquid crystal dispersed with gold and silver nanoparticles. Liquid Crystals, 2015, vol. 42, pp. 1580-1590.
5. Kopčanský P., Tomašovičová N., Koneracká M., Závišová V., Timko M., Džarová A., Šprincová A., Éber N., Fodor-Csorba K., Tóth-Katona T., Vajda A., Jadzyn J. Structural changes in the 6CHBT liquid crystal doped with spherical, rodlike, and chainlike magnetic particles. Physical Review, 2008, vol. 78, 011702.

6. Zakhlevnykh A. N., Petrov D. A.. Weak coupling effects and re-entrant transitions in ferronematic liquid crystals. Journal of Molecular Liquids, 2014, vol. 198, pp. 223-233.

7. Kopčanský P., Tomašovičová N., Tóth-Katona T., Éber N., Timko M, Závišová V., Majorošová J., Rajňak M., Jadzyn J., Chaud X. Increasing the magnetic sensitivity of liquid crystals by rod-like magnetic nanoparticles. Magnetohydrodynamics, 2013, vol. 49, pp. 586-591.

8. Cognard J. Alignment of nematic liquid crystals and their mixtures. London, New York, Paris: Gordon and Breach Sci. Publ. 1982. 104 p.

9. Simon R., Nicholas D. V. Interferometric method of measuring tilt angles in aligned thin films of nematic liquid crystals. Journal of Physics D: Appled Physics, 1985, vol. 18, pp. 1423-1430.

Просьба ссылаться на эту статью в русскоязычных источниках следующим образом:

Пермякова О. О., Попов В. А., Гилев В. Г. Экспериментальное исследование магнитных свойств жидкого кристалла МВВА и ферромагнитных суспензий на его основе // Вестник Пермского университета. Физика. 2017. № 3 (37). С. 60-65. doi: 10.17072/1994-3598-2017-3-60-65

\section{Please cite this article in English as:}

Permyakova O. O., Popov V. A., Gilev V. G. Experimental investigation of the magnetic properties of a liquid crystal MBBA and ferromagnetic suspensions based on it // Bulletin of Perm University. Physics, 2017 , no. 3 (37), pp. 60-65. doi:10.17072/1994-3598-2017-3-60-65 